\title{
Modification of Attenuation Rate in Range Normalization of Echo Levels for Obtaining Frequency-Dependent Intensity Data from 0.6 MHz and 1.0 MHz Devices
}

\author{
Poerbandono \& Totok Suprijo \\ Faculty of Earth Sciences and Technology, Bandung Institute of Technology, \\ Jalan Ganesha No. 10 Bandung 40132, Indonesia \\ Email: poerbandono@gd.itb.ac.id
}

\begin{abstract}
This investigation aims to propose an attenuation rate for range normalization of echo amplitudes recorded by an acoustic backscattering instrument working at a frequency of $1.0 \mathrm{MHz}$. The intention of the use of such an attenuation rate is to obtain equal echo levels when using a device from the same family of products with a different working frequency, i.e. a $0.6 \mathrm{MHz}$ instrument, at an identical site. This work is based on a field experiment with a $1.0 \mathrm{MHz}$ Acoustic Wave and Current (AWAC) profiler and a $0.6 \mathrm{MHz}$ Aquadopp profiler. Both profilers were deployed upward, side-by-side in the Semak Daun reef lagoon, Seribu Islands, Java Sea, Indonesia. It was found that the proposed attenuation rate for the $1.0 \mathrm{MHz}$ instrument was one-order magnitude higher with respect to the one used for the $0.6 \mathrm{MHz}$ instrument, and logarithmically depth dependent. The proposed attenuation rate for the $1.0 \mathrm{MHz}$ AWAC is $-7.925 \log (R)+8.551$, with $R$ is the slant range from the transducers to the measured layer. Accordingly, the overall agreement between the $1.0 \mathrm{MHz}$ AWAC echo amplitude and the one recorded by the $0.6 \mathrm{MHz}$ Aquadopp was improved by $18 \mathrm{~dB}$, which is quite significant considering that the average echo amplitude discrepancy recorded by each transducer was $2.4 \mathrm{~dB}$.
\end{abstract}

Keywords: acoustic backscatter; doppler-type hydro-acoustic current profiler; field deployment; propagation loss; rate of attenuation.

\section{Introduction}

The signal amplitude from acoustic backscattering instruments can be used to measure the quantity of sediment suspended in the water column [1-3]. This applies to Doppler-type hydro-acoustic current or equivalent profilers. In addition to providing three-dimensional current vectors in multiple layers across the water column, such devices also record the intensity of backscattered acoustic signals $[4,5]$. Recently, successful application of such a technique has been reported in [6-9]. Prior to the use of the intensity of backscattered acoustic signals for absolute measurement of suspended sediment concentration, calibration must be performed using field samples [10,11]. This is due to the dependency of the relative measure of the sediment quantity given by the

Received August $29^{\text {th }}, 2012$, Revised February $1^{\text {st }}, 2013$, Accepted for publication April $17^{\text {th }}, 2013$.

Copyright @ 2013 Published by ITB Journal Publisher, ISSN: 2337-5779, DOI: 10.5614/j.eng.technol.sci.2013.45.2.3 
acoustic intensity on the device used and properties of the sediment in suspension. Recent field applications of acoustic measurement of suspended sediment have suggested that field calibration is site-specific and frequencydependent $[12,13]$. Furthermore, devices from the same product family using different frequencies do not necessarily give similar echo amplitudes in an identical environment. Apparently, as shown in [14], records of echo amplitude in spatial and temporal series are useful in providing insight into the periodic occurrence of diurnal signals. Hence, improvement of the performance in the relative detection of suspended sediment concentrations from the intensity of acoustic backscatter data is necessary. The objective of this study is to propose an improved term for the normalization of acoustic signal amplitudes. The attenuation rate of SONAR propagation will be adjusted to make it dependent on the devices' working frequency. This adjustment is meant to equalize the reading of the intensity of the backscattered acoustic signals obtained from devices with unequal working frequencies. This will optimize efforts in the broader application of coastal and offshore survey work, particularly for suspended sediment and sediment transport monitoring, since field deployment no longer necessitates the use of devices with equal frequencies. The work presented in this paper is based on a field experiment using Nortek A/S devices from the same family of products.

\section{Material and Method}

\subsection{Acoustic Detection of Water Column Turbidity}

The so-called random phase scattering model assumes direct proportionality between the returning acoustic signal and the volume of backscattering strength $[1,2]$. Since the volume of backscattering strength in the water column is due to the presence of sediment in suspension, empirical calibration for quantification of the suspended sediment concentration on the basis of the intensity of acoustic backscatter can be done as follows $[8,11]$ :

$$
E L \propto 10 \log _{10}(c)
$$

where $E L=$ echo level or intensity of acoustic backscatter $(\mathrm{dB})$ and $c=$ concentration of sediment in suspension (in $\mathrm{kg} / \mathrm{m}^{3}$ ). In order to estimate the absolute quantity of the sediment suspended in the water column, the recorded intensity of the acoustic backscatter (i.e. EL) must undergo scaling, normalization and calibration against field samples. The scaling converts the recorded intensity of the acoustic backscatter from internal units of count to decibels $(\mathrm{dB})$. The normalization makes the recorded values independent from the range and size of sediment particles and instruments used. Calibration is intended to estimate the backscattering strength $(B S)$ that is assumed to be due 
to the presence of sediment in suspension. The underlying empirical SONAR equation reads as [15]:

$$
E L=S L-2 T L+B S
$$

where $E L=K A$ with $K=$ scaling factor and $A=$ recorded signal amplitude in count units, $S L=$ source level or transmitted intensity of acoustic pulse, $T L=$ transmission loss due to (i) geometrical spreading $20 \log (R)$, (ii) attenuation of SONAR propagation due to medium $\alpha R$, and (iii) attenuation due to sediment particles $20 R \int \alpha_{\mathrm{p}} \mathrm{d} r, R=$ slant range or oblique distance from transducer to measured layer, $\alpha=$ attenuation due to medium, and $\alpha_{\mathrm{p}}=$ attenuation due to sediment particles. Figure 1 illustrates the terms used in Eq. (2), $\varphi$ is the angle between vertical and transducer axis. $R$ must be calculated from the vertical distance $(D)$ between the measured layer and the face of the transducer (see Figure 1).

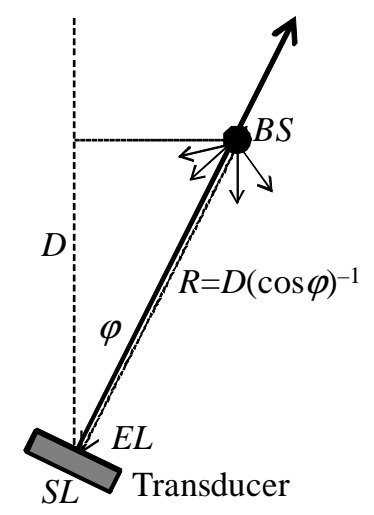

Figure 1 Schematic illustration of SONAR propagation.

For devices from the Nortek A/S product family as used here, the suggested $K$ is 0.43 [5]. In low-concentration environments, attenuation due to sediment particles can be neglected and with the use of similar devices at an identical site, the proportionality between echo level and backscattering strength is scaled by the attenuation rate due to medium $(\alpha)$.

\subsection{Field Experiment and Data Analysis}

Two Nortek A/S products were simultaneously deployed: a $1.0 \mathrm{MHz}$ Acoustic Wave And Current (AWAC) profiler (S/N WPR 0677), and a $0.6 \mathrm{MHz}$ Aquadopp profiler (S/N AQD 5775), looking upward from the seabed, at a depth of roughly 11.5 meters. The devices were separately fixed in their mounting frames (Figure 2) and weighted during deployment. The lowering of the devices to the seabed was done manually with the help of scuba divers. Prior 
to this, the points of deployment were marked by moored floating buoys at an approximate distance of $11 \mathrm{~m}$ (Figure 3). The site of the experiment was located in a lagoon of the Semak Daun reef platform of Seribu Islands off Jakarta Bay, Southwest Java Sea, Indonesia. The deployment lasted for approximately 40 hours, starting on the 15th of April, 2011 at 16:15 and ending on the 17th of April, 2011 at 08:30. The recording was set at a 15-minute interval with an average interval per data acquisition of 60 seconds. Throughout the deployment, as many as 162 time series data sets were collected from each device, at each vertical layer (out of eight effective layers).

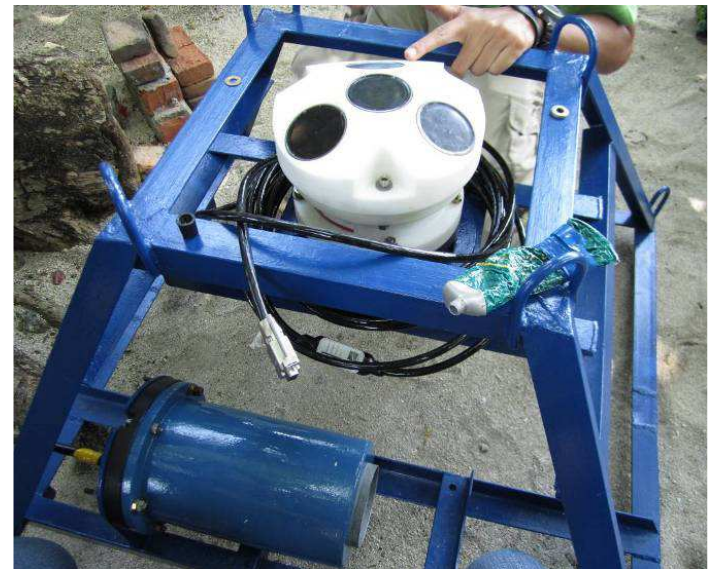

(a) $1.0 \mathrm{MHz}$ AWAC

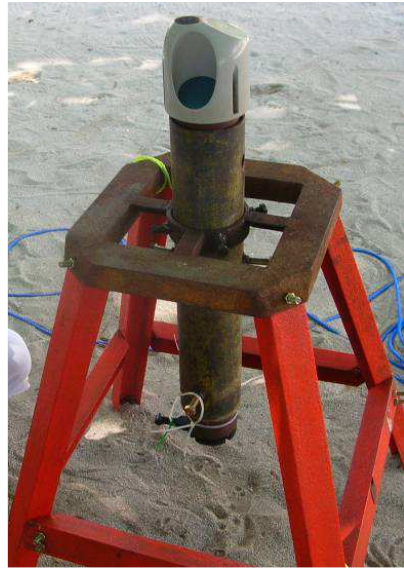

(b) $0.6 \mathrm{MHz}$ Aquadopp

Figure 2 Doppler-type acoustic current profilers (Photo: Miga M. Julian).

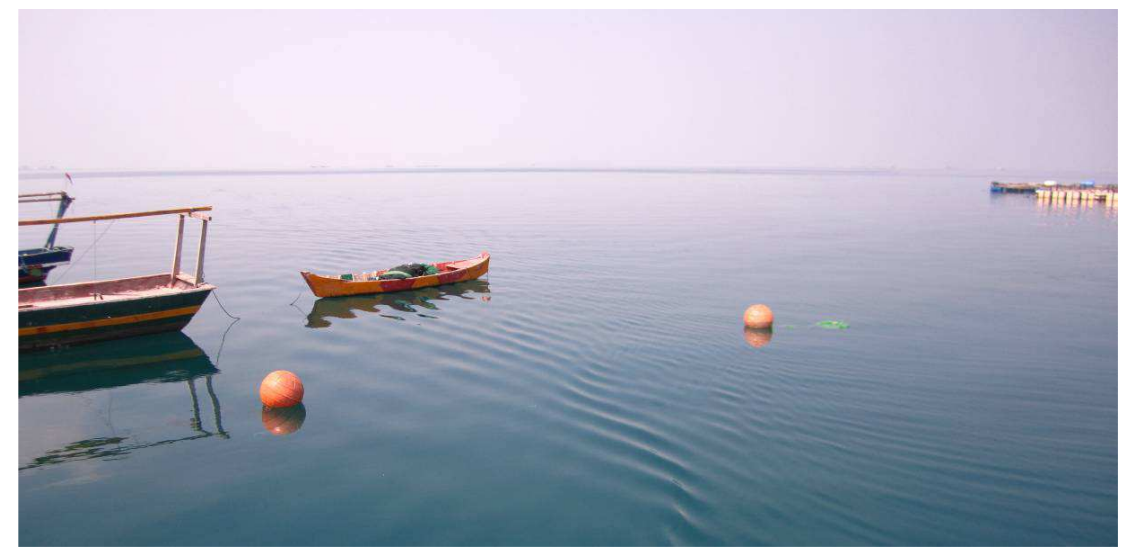

Figure 3 Marker buoys indicating seabed mounted devices at the deployment site (Photo: Miga M. Julian). 
The analysis of the data involved quality control of measured parameters and characterization of the recorded intensity of the acoustic backscatter. Further to this, determination of the rate of attenuation for the $1.0 \mathrm{MHz}$ AWAC was done by assuming a fixed rate of attenuation for the $0.6 \mathrm{MHz}$ instrument $\left(\alpha_{0.6}\right)$. For this particular purpose a value documented in [5] was used, i.e. $\alpha_{0.6}=0.15 \mathrm{~dB} / \mathrm{m}$. For the initial approach, a suggested value was also used for the $1.0 \mathrm{MHz}$ instrument, i.e. $\alpha_{1.0}{ }^{*}=0.4 \mathrm{~dB} / \mathrm{m}$. The corresponding results were evaluated according to the absolute discrepancy $(e)$ between the echo levels recorded by the $1.0 \mathrm{MHz}$ device and the $0.6 \mathrm{MHz}$ device:

$$
e(t, z)=\left|E L_{1.0}(t, z)-E L_{0.6}(t, z)\right|
$$

where $E L_{0.6}=K A_{0.6}+40 \log (R)+2 \alpha_{0.6} R$ and $E L_{1.0}=K A_{1.0}+40 \log (R)+$ $2 k \alpha_{1.0} * R$, with $t=$ time, $z=$ depth or height of measured layer and $k=$ modification factor, which was determined empirically here. This procedure was applied to each of the measuring layers.

\section{Result and Discussion}

\subsection{Field Records and Data Pre-Processing}

Figure 4 shows the log of sound speed, water level, temperature, heading, pitch and roll of each device. Sudden changes in values at the very beginning and end of the time series plots in Figure 4 indicate deployment and recovery. This indicates that both devices were aligned well, without significant movement, and experienced quite a steady physical environment during observation. Figure 5 shows temporal (over the observation period) and spatial (throughout the measured water column) series plots of the echo level after scaling and default range normalization as proposed by [5]. One may observe that the $1.0 \mathrm{MHz}$ AWAC device generally provided underestimated values compared to those provided by the $0.6 \mathrm{MHz}$ Aquadopp device.

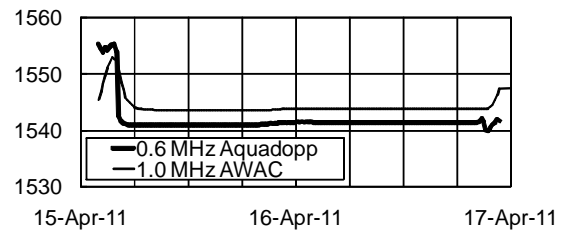

(a) Sound speed in $\mathrm{m} / \mathrm{s}$

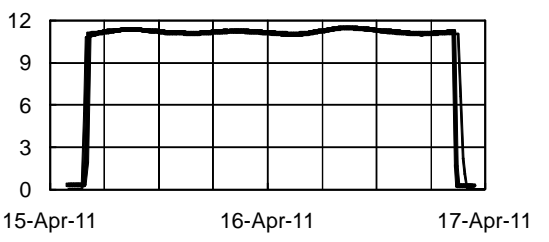

(b) Water level in $\mathrm{m}$

Figure 4 Field records of water properties (a, b, c) and sensor alignment (d, e, f). 


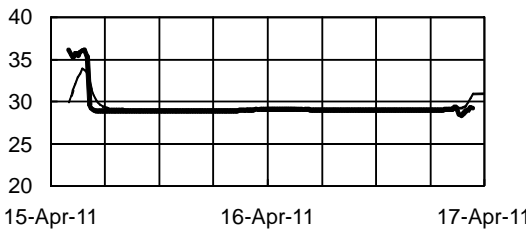

(c) Temperature in ${ }^{\circ} \mathrm{C}$

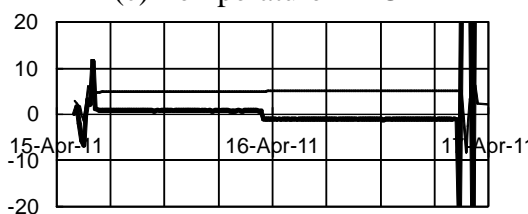

(e) Pitch in degree

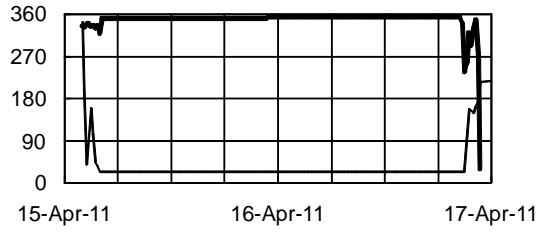

(d) Heading in degree

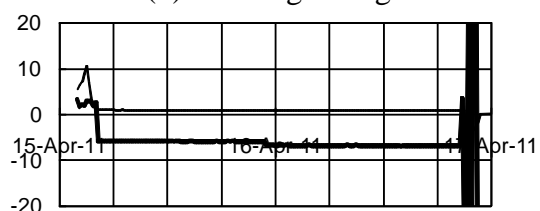

(f) Roll in degree

Figure 4 Continued. Field records of water properties (a, b, c) and sensor alignment (d, e, f).

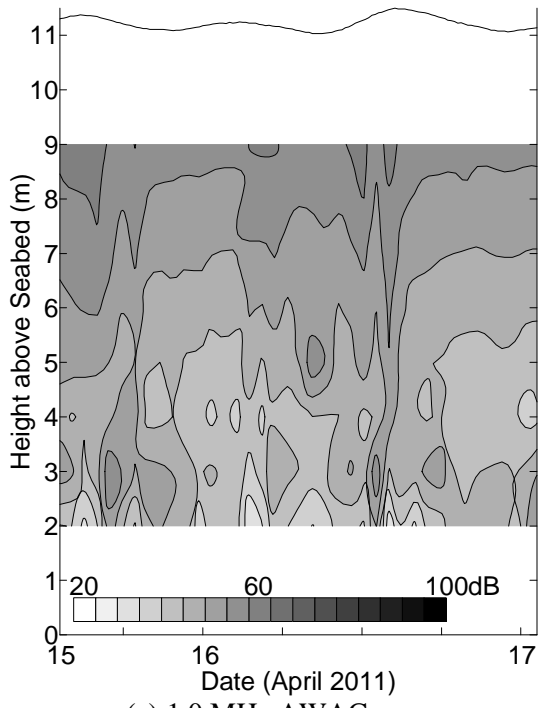

(a) $1.0 \mathrm{MHz}$ AWAC.

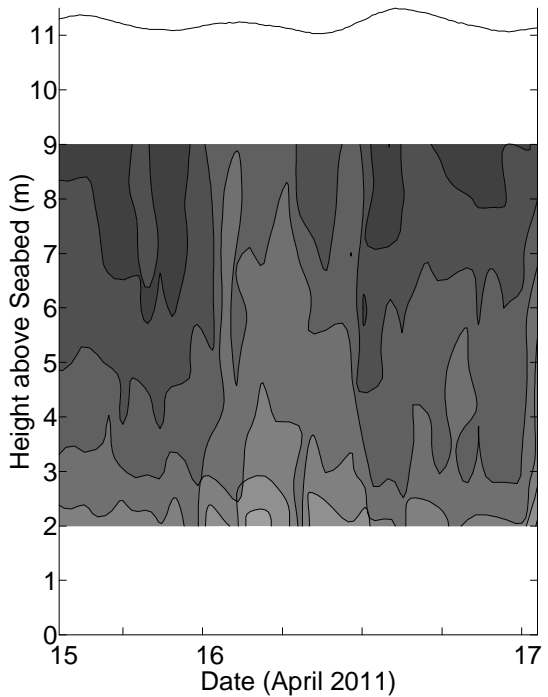

(b) $0.6 \mathrm{MHz}$ Aquadopp.

Figure 5 Echo level over water column and observation period in $\mathrm{dB}$.

\subsection{Assessment of Agreement and Correction of Rate of Attenuation}

The agreement measure of the acoustic strength between the $1.0 \mathrm{MHz}$ AWAC and the $0.6 \mathrm{MHz}$ Aquadopp was calculated according to Eq. (3) and the layerby-layer results, with an overall average agreement $(\bar{e}(t, w))$ of $22.2 \mathrm{~dB}$. The results are displayed in Table 1 . The absolute discrepancy between the intensities recorded by both transducers was also evaluated. This was done by 
comparing the magnitudes recorded by transducers III and II to those recorded by transducer I. The corresponding results are shown in Table 2 and give an overall average of $2.4 \mathrm{~dB}$.

Table 1 Average agreement of acoustic strengths between layers in dB.

\begin{tabular}{lcccccccc} 
& \multicolumn{7}{c}{ Layer \# } \\
\cline { 2 - 9 } & $\mathbf{1}$ & $\mathbf{2}$ & $\mathbf{3}$ & $\mathbf{4}$ & $\mathbf{5}$ & $\mathbf{6}$ & $\mathbf{7}$ & $\mathbf{8}$ \\
\hline$R(\mathrm{~m})$ & 1.1 & 2.2 & 3.3 & 4.4 & 5.5 & 6.6 & 7.7 & 8.8 \\
Mean $E L$ of $1.0 \mathrm{MHz}$ & 42 & 48 & 44 & 47 & 50 & 53 & 56 & 58 \\
Mean $E L$ of $0.6 \mathrm{MHz}$ & 61 & 68 & 71 & 72 & 74 & 76 & 77 & 79 \\
$\bar{e}(t, z)$ & 18 & 20 & 26 & 25 & 24 & 23 & 22 & 20 \\
\hline
\end{tabular}

Table 2 Overall average of agreement of acoustic strengths between transducers.

\begin{tabular}{cccc}
\hline \multicolumn{2}{c}{ 1.0 MHz AWAC } & \multicolumn{2}{c}{ 0.6 MHz Aquadopp } \\
\hline II to I & III to I & II to I & III to I \\
\hline $2.2 \mathrm{~dB}$ & $2.0 \mathrm{~dB}$ & $3.0 \mathrm{~dB}$ & $2.4 \mathrm{~dB}$ \\
\hline
\end{tabular}

The discrepancy between the echo levels provided by the $1.0 \mathrm{MHz}$ AWAC and the $0.6 \mathrm{MHz}$ Aquadopp is one-order of magnitude greater than the variability of the intensity reading in each transducer. Hence, the normalization of the echo level of the $1.0 \mathrm{MHz}$ AWAC was intervened by modifying $k$ (see: Eq. (3) and the corresponding consecutive description) in such a way that the discrepancy was smallest, and this procedure was applied to each measuring layer. The results are given in Table 3 as $\bar{e}(t, z)$ in $\mathrm{dB}$. The corresponding overall average of the absolute discrepancy was found to be $4.3 \mathrm{~dB}$. Hence, the suggested rate of attenuation for the $1.0 \mathrm{MHz}$ AWAC is proposed in Table 3 as $\alpha_{1.0}{ }^{* *}$, where $\alpha_{1.0}{ }^{* *}=k \alpha_{1.0} *$ in $\mathrm{dB} / \mathrm{m}$. From Table 3, one can see that the applied rate of attenuation for each measuring layer is not uniform. This will be discussed in the subsequent section.

Table 3 Proposed layer-specific attenuation rate for $1.0 \mathrm{MHz}$ AWAC, i.e. $\alpha_{1.0} * *$.

\begin{tabular}{lcccccccc} 
& \multicolumn{8}{c}{ Layer \# } \\
\cline { 2 - 9 } & $\mathbf{1}$ & $\mathbf{2}$ & $\mathbf{3}$ & $\mathbf{4}$ & $\mathbf{5}$ & $\mathbf{6}$ & $\mathbf{7}$ & $\mathbf{8}$ \\
\hline$R(\mathrm{~m})$ & 1.1 & 2.2 & 3.3 & 4.4 & 5.5 & 6.6 & 7.7 & 8.8 \\
$k(-)$ & 23 & 13 & 11 & 8 & 6.5 & 5.3 & 4.5 & 3.8 \\
$\bar{e}(t, z)(\mathrm{dB})$ & 5.7 & 4.3 & 3.8 & 4.0 & 3.7 & 4.0 & 4.1 & 4.5 \\
$\alpha_{1.0} * *(\mathrm{~dB} / \mathrm{m})$ & 9.0 & 5.0 & 4.3 & 3.2 & 2.6 & 2.1 & 1.8 & 1.5 \\
\hline Note: $\alpha_{1.0} * *=k \alpha_{1.0} *$ & $\alpha_{1.0} *$ & $=0.4 \mathrm{~dB} / \mathrm{m}$
\end{tabular}

The effects of using a modified constant as the rate of attenuation for each layer of the recorded backscatter intensity from the $1.0 \mathrm{MHz}$ AWAC data, can be seen from Figures 6-7. In Figure 6(a), the acoustic backscatter intensity recorded by 
the 1.0 MHz AWAC (after modification of its rate of attenuation) is shown as a plot of the spatial and temporal data series. Referring back to Figure 5(a) for an impression of the initial results, the spatio-temporal agreement between the acoustic backscatter intensity of the $1.0 \mathrm{MHz}$ AWAC (after modification of its rate of attenuation) and the $0.6 \mathrm{MHz}$ Aquadopp is given in Figure 6(b). Figure 7 shows time series plots of the intensity of the acoustic backscatter for each layer, as recorded by the $0.6 \mathrm{MHz}$ Aquadopp and the $1.0 \mathrm{MHz}$ AWAC respectively, before and after modification of the attenuation rate.

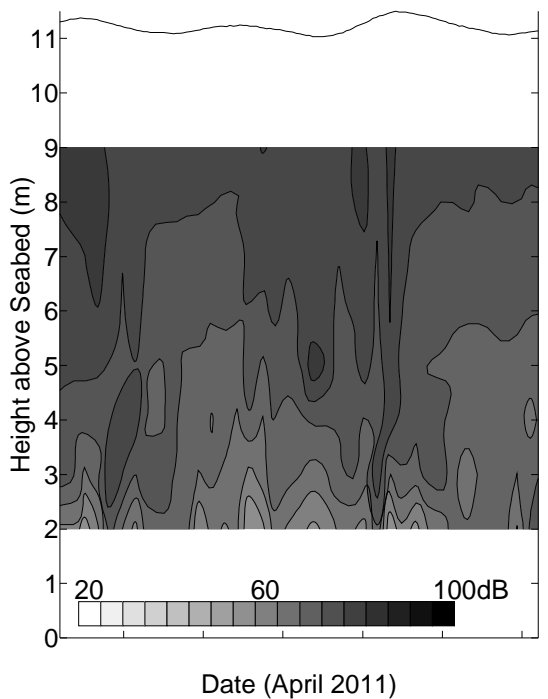

(a) $1.0 \mathrm{MHz}$ AWAC.

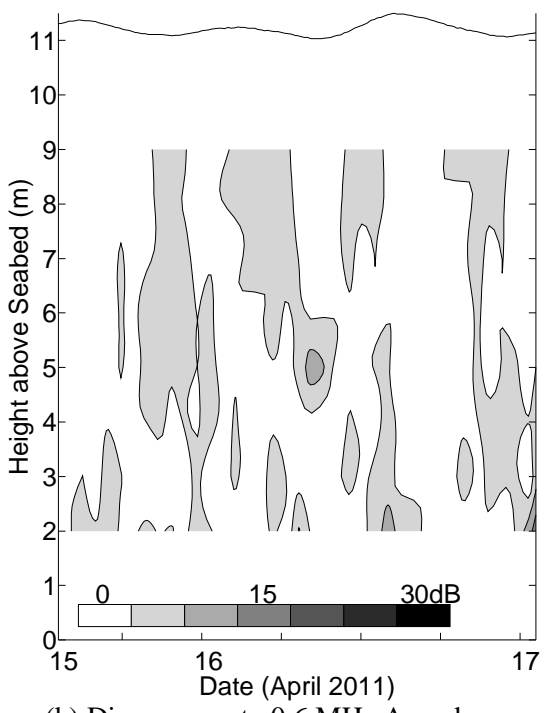

(b) Discrepancy to $0.6 \mathrm{MHz}$ Aquadopp.

Figure 6 Echo level of $1.0 \mathrm{MHz} \mathrm{AWAC}$ and agreement in $\mathrm{dB}$.

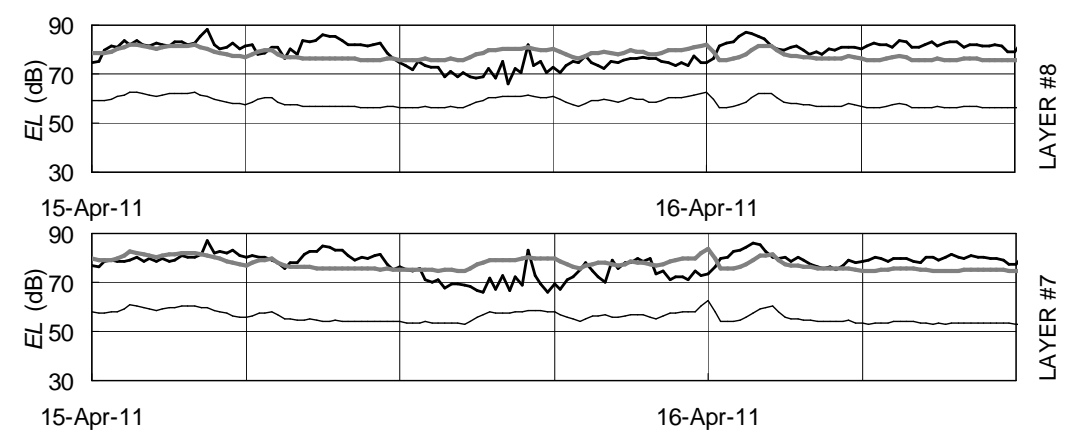

Figure 7 Temporal changes of range-normalized echo level (EL). 

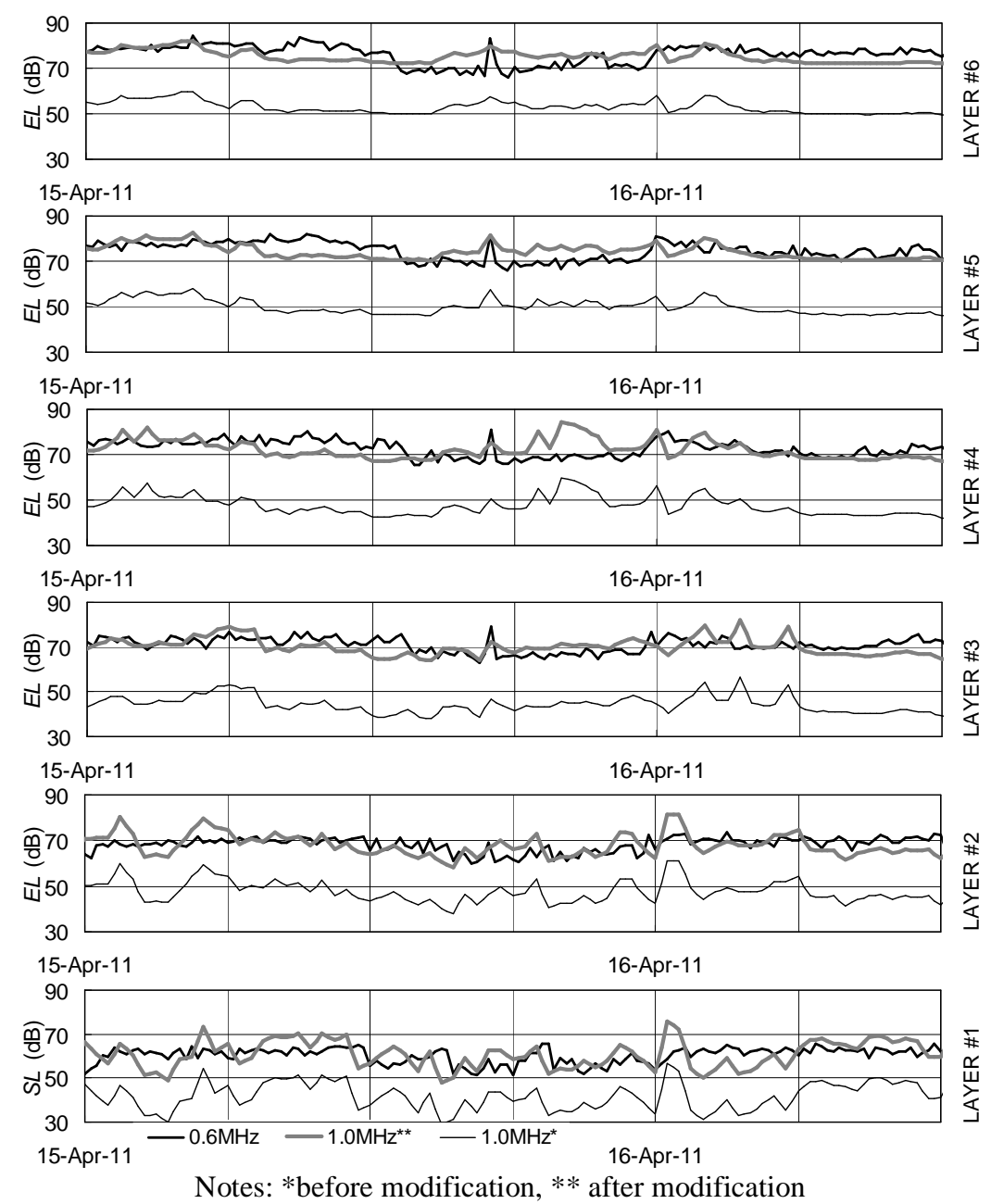

Figure 7 Continued. Temporal changes of range-normalized echo level (EL).

\subsection{Relation between Range and Rate of Attenuation}

In order to deliver backscatter intensity data from the $1.0 \mathrm{MHz}$ AWAC that fit best with those recorded by the $0.6 \mathrm{MHz}$ Aquadopp, to each of the measuring layers a proposed rate of attenuation for the $1.0 \mathrm{MHz}$ AWAC must be applied separately, and, hence, the rate of attenuation is range-dependent. A further analysis was made and it was indicated that there was a proportional relation between the range from the transducers' faces to the measured layer (i.e. slant range $-R$, see: Figure 1) and the applied rate of attenuation. The relation on a 
logarithmic scale between the slant range $(R)$ and the proposed rate of attenuation for the 1.0 MHz AWAC is shown in Figure 8. A strong correlation is seen with the coefficient of determination $\left(r^{2}\right)$ of 0.958 . We therefore propose to use a modified rate of attenuation for the $1.0 \mathrm{MHz}$ AWAC as $\alpha_{1.0}{ }^{* *}=$ $-7.925 \log (R)+8.551$ in order to find a fit with the backscatter intensity data recorded by $0.6 \mathrm{MHz}$ Aquadopp.

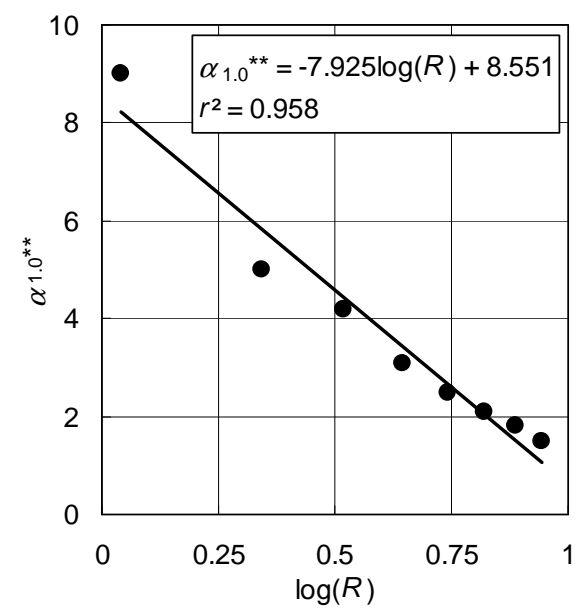

Figure 8 Proposed range-dependant rate of attenuation for the $1.0 \mathrm{MHz}$ AWAC.

\subsection{Accuracy and Limitation}

Previous field tests with similar devices (1.0 MHz system) in the same area suggested that the overall accuracy of acoustic detection of suspended sediment concentrations using backscatter intensity data is within a factor of $2[12,13]$. This was done by developing a calibration curve in which echo levels and sampled concentrations of suspended sediment are related to each other on a logarithmic scale. In Figure 9, calibration curves for estimating the suspended sediment concentration from recorded backscatter intensity as reported in [16] are given.

The calibration curves and scatters of echo levels and suspended sediment concentration data as shown in Figure 9, suggest that there is a trend of increasing echo levels with an increase of the concentration of suspended sediment. On an equal range of echo levels and using a similar device (i.e. with a $1.0 \mathrm{MHz}$ working frequency), different sites provide a dissimilar order of magnitude of sampled concentrations of suspended sediment. One may see from Figure 9 that in the range of echo levels between $60 \mathrm{~dB}$ and $80 \mathrm{~dB}$, a large 
difference in the logarithmic expression of the suspended sediment concentration was observed. This is due to the dissimilar characteristics of the acoustic strengths scattered back to the devices' transducers from inhomogeneous physical properties of the suspended sediment. Unless direct samples of suspended sediment are collected simultaneously with acoustic profiling, no direct relation between echo levels and suspended sediment concentration data can be described.

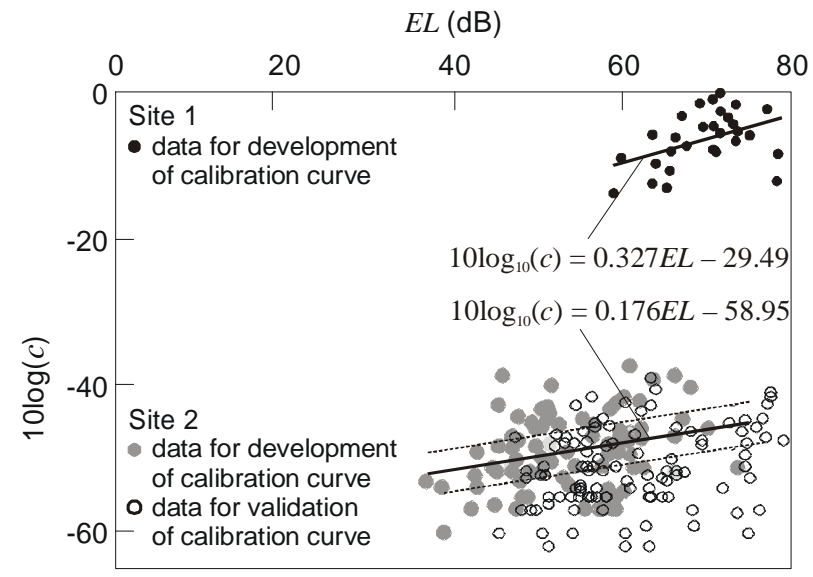

Figure 9 Calibration curves for estimating suspended sediment concentration from recorded backscatter intensity [modified from 16].

The objective of this study was to normalize data from two devices with different working frequencies (i.e. $1.0 \mathrm{MHz}$ and $0.6 \mathrm{MHz}$ ) in view of delivering comparable magnitudes of backscatter intensity. A further assessment of the absolute accuracy, which could be obtained from applying the modified rate of attenuation as proposed in this paper, is still wanted. This would require auxiliary field experiments along with a thorough analysis of the field samples. Additionally, the applied field experiment presented here must be understood to work under the assumption that the physical properties of the sediment in suspension are uniform. Field applications at different locations with a similar set of devices' working frequencies (i.e. $1.0 \mathrm{MHz}$ and $0.6 \mathrm{MHz}$ as used here) must be executed to ensure that the physical properties of the suspended sediment detected by each device are uniform.

\section{Conclusion}

The rate of attenuation proposed for the $1.0 \mathrm{MHz}$ AWAC profiler is found to be one-order of magnitude higher compared to that used by the $0.6 \mathrm{MHz}$ Aquadopp profiler and logarithmically range-dependent. The proposed 
attenuation rate for the $1.0 \mathrm{MHz}$ device is $-7.925 \log (R)+8.551$, with $R$ is the slant range from the transducers to the measured layer. The use of the proposed rate of attenuation as presented in this paper has improved the overall agreement between the $1.0 \mathrm{MHz}$ device's echo level and the one recorded by the $0.6 \mathrm{MHz}$ instrument from $22 \mathrm{~dB}$ to $4 \mathrm{~dB}$. This is considered quite significant, bearing in mind that the average discrepancy between the echo levels recorded by each transducer was $2.4 \mathrm{~dB}$. With the use of a default rate of attenuation of $0.15 \mathrm{~dB} / \mathrm{m}$ for the $0.6 \mathrm{MHz}$ instrument, comparable magnitudes of acoustic backscatter can also be obtained by the $1.0 \mathrm{MHz}$ instrument, applying the rate of attenuation proposed in this paper.

\section{Acknowledgements}

This research was funded by ITB Research Scheme 2011. The authors acknowledge Mr. Miga M. Julian and Mr. Andhika S. Putra for their help during field deployment. Acknowledgement is also addressed to Mr. Sri W. Waskito for the supervisory work during the scuba diving. The authors also thank PT. Hidronav Teknikatama Jakarta for their device support. This submission has benefited from two anonymous reviewers.

\section{References}

[1] Thorne, P.D., Hardcastle, P.J. \& Soulsby, R.L., Analysis of Acoustic Measurements of Suspended Sediments, Journal of Geophysical Research, 98(C1), pp. 899-910, 1993.

[2] Deines, K.L., Backscatter Estimation Using Broadband Acoustic Doppler Current Profilers, In: Anderson, S.P., Terry, E.A., White, J.A.R., William, A.J., eds., Proceedings of IEEE 6th Working Conference on Current Measurement Technology, pp. 249-253, 1999.

[3] Holdaway, G.P., Thorne, P.D., Flatt, D., Jones, S.E. \& Prandle, D., Comparison between ADCP and Transmissometer Measurements of Suspended Sediment Concentration, Continental Shelf Research, 19(3), pp. 421-441, 1999.

[4] Gordon, R.L., Acoustic Doppler Current Profiler: Principles of Operation, RD Instruments, San Diego, USA, $2^{\text {nd }}$ edition, 1996.

[5] Lohrmann, A., Monitoring Sediment Concentration with Acoustic Backscattering Instruments, Nortek Technical Note, Number 003, pp. 5, 2001.

[6] Ghaffari, P., Azizpour, J., Noranian, M., Chegini, V., Tavakoli, V. \& ShahHosseini, M., Estimating Suspended Sediment Concentrations Using A Broadband ADCP in Mahshahr Tidal Channel, Ocean Science Discussion 8, pp. 1601-1630, 2011. 
[7] Merckelbach, L.M., A Model for High-Frequency Acoustic Doppler Current Profiler Backscatter from Suspended Sediment in Strong Currents, Continental Shelf Research, 26, pp. 1316-1335, 2006.

[8] Poerbandono \& Mayerle, R., Effectiveness of Acoustic Profiling for Estimating the Concentration of Suspended Material, Die Küste, 69, pp. 393-407, 2005.

[9] Kostaschuk, R., Best, J., Villard, P., Peakall, J., Franklin, M., Measuring Flow Velocity and Sediment Transport with an Acoustic Doppler Current Profiler, Geomorphology, 68(1-2), pp. 25-37, 2005.

[10] Poerbandono, Sediment Transport Measurements and Modeling in The Meldorf Bight Tidal Channels, German North Sea Coast, Dissertation, Christian-Albrechts-Universität zu Kiel, 2003.

[11] Gartner, J.W., Estimating Suspended Solids Concentrations from Backscatter Intensity Measured by Acoustic Doppler Current Profiler in San Francisco Bay, California, Marine Geology, 211, pp. 169-187, 2004.

[12] Poerbandono \& Magetsari R.N., Identification of Representative Erosion and Accretion Patterns Across North Java Coasts on The Basis of Analytical Study of Current and Seabed Interaction, Report, Foundation for Research and Community Services, Institut Teknologi Bandung, 2007.

[13] Poerbandono \& Mirza, A., Sensitivity and Accuracy Assessments of Doppler-Type Hydro-Acoustic Current Profilers for Estimating Suspended Particulate Matter Concentration from Field Tests, Proceedings of the $10^{\text {th }}$ SE Asian Survey Congress, Nusa Dua, Bali, 4-7 August 2009.

[14] Kaneda, A., Takeoka, H., Koizumi, Y., Periodic Occurrence of Diurnal Signal of ADCP Backscatter Strength in Uchiumi Bay, Japan, Estuarine, Coastal and Shelf Science, 55, pp. 323-330, 2002.

[15] Lurton, X., An Introduction to Underwater Acoustics: Principles and Applications, Praxis Publishing, Chichester, UK, 2002.

[16] Poerbandono, On the Interpretation of Suspended Sediment Concentration from Acoustic Backscattering Amplitude for Sediment Flux Studies, Proceedings of the $11^{\text {th }}$ International Symposium and Exhibition on Geoinformation, Kuala Lumpur, 25-26 September 2012. 